\title{
Depressão e ansiedade nos cuidadores de mulheres em fase terminal de câncer de mama e ginecologicico
}

\author{
Depression and anxiety in caregivers of terminally-ill breast and gynecological cancer patients
}

Vera Lucia Rezende ${ }^{1}$, Sophie Françoise Mauricette Derchain ${ }^{2}$, Neury José Botega ${ }^{3}$, Luis Otávio Sarian ${ }^{4}$, Daniela Landulfo Vial ${ }^{5}$, Sirlei Siani Morais ${ }^{6}$

\section{RESUMO}

Objetivo: avaliar a freqüencia de ansiedade e depressão em cuidadores principais de mulheres em fase terminal de câncer de mama ou genital. Métodos: para este estudo de corte transversal foram incluídos 133 cuidadores de pacientes sem possibilidades curativas, internadas no Centro de Atenção Integral à Saúde da Mulher, entre agosto de 2002 e maio de 2004. Das pacientes incluídas, 71 apresentavam câncer de mama e 62, câncer ginecológico. Foi aplicada a Escala Hospitalar de Ansiedade e Depressão e realizada entrevista para obter outras informações como idade, sexo, religião, parentesco com a paciente, profissão, se cuidava de outras pessoas, se a rotina dele mudou e se outras pessoas ajudavam a cuidar da paciente. Utilizou-se a regressão logística para cálculo do odds ratio (OR) e seus respectivos intervalos de confiança (IC), para avaliar a relação entre os diagnósticos de ansiedade e depressão entre os cuidadores informais. Para a análise múltipla foi considerado o critério de seleção de variáveis passo a passo. Resultados: observou-se que $43 \%$ das pacientes indicaram como cuidador principal a filha e $24 \%$ o marido. A maioria dos cuidadores tinha idade superior a 35 anos (63\%), $68 \%$ eram do sexo feminino, $59 \%$ estavam desempregados ou aposentados, $47 \%$ cuidavam de outra pessoa e $84 \%$ referiram mudança na rotina pelo fato de cuidar. A ansiedade foi detectada em 99 cuidadores principais $(74,4 \%)$ e a depressão em 71 (53,4\%), sendo estes estados fortemente relacionados entre si ( $\mathrm{OR}=5,6$; IC 95\%: 2,2 a 15,9). Na análise bivariada, o marido apresentou menos ansiedade e, após regressão logística, apenas o fato de ser homem esteve relacionado com menor ansiedade. Conclusão: o processo de cuidar de paciente na fase terminal levou a altas taxas de ansiedade e depressão. Os homens e maridos despontaram neste estudo como cuidadores menos ansiosos.

PALAVRAS-CHAVE: Qualidade de vida; Assistência paliativa; Neoplasias mamárias; Neoplasias dos genitais femininos; Depressão; Ansiedade

\section{ABSTRACT}

Purpose: to evaluate the frequency of anxiety and depression in main caretakers of patients with terminal breast or gynecological cancer. Methods: for this cross-sectional study, 133 informal caretakers of terminally-ill breast and gynecologic cancer patients were included. Patients were hospitalized for palliative care in the Oncology Clinic of the "Centro de Atenção Integral à Saúde da Mulher (Campinas, Brasil) from August 2002 to May 2004. Seventy-one of the patients had breast cancer and 62 had gynecological malignancies. Hospital Anxiety and Depression Scale (HAD) was applied to these informal caretakers, in order to detect anxiety and depression, and they were also interviewed to provide additional information regarding their age, gender, religion, relation to patient, current occupation, if they cared for other people, whether their routine had changed and whether other people helped them to care for the patient. Logistic regression was used to calculate the odds ratio (OR) and its confidence interval (CI), used to assess the relationship between the diagnoses of anxiety and depression among the informal caretakers. For multiple analyses, the stepwise criterion for variable selection was used.

1 Psicóloga, Mestre em Saúde Mental, Doutora em Tocoginecologia, Centro de Atenção Integral à Saúde da Mulher (CAISM). Universidade Estadual de Campinas - UNICAMP - Campinas (SP) - Brasil.

2 Professora Associada do Departamento de Tocoginecologia, Faculdade de Ciências Médicas, Centro de Atenção Integral à Saúde da Mulher (CAISM), Universidade Estadual de Campinas - UNICAMP - Campinas (SP) - Brasil.

3 Professor Titular do Departamento de Psicologia Médica e Psiquiatria, Faculdade de Ciências Médicas, Universidade Estadual de Campinas - UNICAMP - Campinas (SP) - Brasil.

4 Doutor em Tocoginecologia, Departamento de Tocoginecologia, Faculdade de Ciências Médicas, Centro de Atenção Integral à Saúde da Mulher (CAISM) Universidade Estadual de Campinas - UNICAMP - Campinas (SP) - Brasil.

5 Psicóloga do Centro de Atenção Integral à Saúde da Mulher (CAISM), Universidade Estadual de Campinas - UNICAMP - Campinas (SP) - Brasil.

6 Estatística do Centro de Atenção Integral à Saúde da Mulher (CAISM), Universidade Estadual de Campinas - UNICAMP - Campinas (SP) - Brasil.

Correspondência: Sophie FM Derchain

Rua Dr António Hossri, 629 - Cidade Universitária - 13 083-370 - Campinas - SP - Telefone: 55 (19) $3788-9305 / 55$ (19) $3287-5538$ - e-mail: derchain@fcm.unicamp.br 
Results: $43 \%$ percent of the patients identified their daughters as their main caretaker, and 24\%, their husbands. Most of the caretakers were over 35 years old (63\%), 68\% were female, 59\% were unemployed, $47 \%$ cared for another person and $84 \%$ referred that his/her routine had changed because of caring. Anxiety was detected in 99 caretakers (74.4\%) and depression in $71(53.4 \%)$. Anxiety and depression were strongly correlated (odds ratio 5.6; 95\% confidence interval 2.2 to 15.9). Bivariate analysis disclosed that the patients' husbands were less affected by depression, but multivariate analysis revealed that only the fact of being male was related to a lower prevalence of anxiety. Conclusion: caring for terminally-ill cancer patients led to high prevalence of anxiety and depression. Only men and the patients' husbands were found to have a lower prevalence of anxiety.

KEYWORDS: Quality of life; Palliative care; Breast neoplasms; Genital neoplasms, female; Depression; Anxiety

\section{Introdução}

Doentes na fase final do câncer encontramse freqüentemente debilitados e dependentes. A dor, confusão mental, dificuldades respiratórias, alimentares e de locomoção, assim como ansiedade e depressão são os problemas mais comumente relatados ${ }^{1}$. É nesta fase que o papel do cuidador se torna altamente relevante. Um dos objetivos dos cuidados paliativos é a atenção às necessidades destes cuidadores informais, em geral parentes ou amigos destas pacientes com câncer em fase terminal ${ }^{2-4}$.

Cuidar significa dedicar longos períodos de tempo ao paciente, somado ao desgaste físico, custos financeiros, sobrecarga emocional e exposição a riscos mentais e físicos ${ }^{5}$. A maioria dos trabalhos tem avaliado familiares no período de luto, pouco se conhecendo sobre o estado psicológico do cuidador na fase que antecede a morte ${ }^{6}$. Há três grandes categorias de demandas relacionadas ao cuidador informal: sobrecarga, necessidades e qualidade de vida. A sobrecarga pode ser objetiva ou subjetiva: a objetiva corresponde às demandas no papel do cuidar propriamente dito e a subjetiva se relaciona às sensações que o ato de cuidar desencadeia. As necessidades são agrupadas em cognitivas, emocionais e fisicas ${ }^{7}$. Finalmente, a qualidade de vida é definida como bem-estar físico, emocional e social ${ }^{8}$.

Pouco se conhece sobre o estado psicológico do cuidador principal no período que antecede a morte do paciente. Estudo recente encontrou altos níveis de ansiedade em cuidadores primários de pacientes do sexo masculino (45\%), como também em cuidadores de pacientes do sexo feminino $(47 \%)^{9}$. A depressão é mais estudada e descrita em cerca de $30 \%$ dos cuidadores, sendo mais prevalente em cuidadores de pacientes do sexo feminino $^{9-12}$. Verifica-se também aumento progressivo desses sintomas conforme a aproximação do óbito ${ }^{6}$.
A ansiedade define um estado de alerta, que amplia o estado de atenção diante de uma situação de perigo real ou imaginário. Está presente como uma sensação difusa, desagradável, de apreensão, acompanhada por várias sensações físicas como mal-estar gástrico, precordialgia, palpitações, sudorese excessiva e cefaléia ${ }^{12}$. Os sintomas depressivos podem se manifestar de várias formas. As alterações de humor podem ser tristeza, perda de interesse por qualquer atividade, falta de prazer, crises de choro ou variação diurna do humor. As alterações motoras incluem inibição ou retardo dos movimentos e agitação, principalmente em idosos. As mudanças somáticas aparecem como alterações no sono, tais como insônia ou hipersonia, variações do apetite e peso, perda de libido, obstipação e fadiga. As sociais incluem apatia, isolamento e incapacitação para o desempenho das tarefas cotidianas. As alterações cognitivas relacionam-se com desesperança, desamparo, idéias de culpa e de suicídio, indecisão e perda de insight, isto é, do reconhecimento de que está doente. Sintomas de ansiedade, tanto psíquica como somática e fóbica, podem aparecer, bem como sintomas de irritabilidade, que podem se manifestar na forma de hostilidade, auto e heterodirigida ${ }^{13}$.

A prevenção da ansiedade e da depressão, tanto do paciente como da família, é mais efetiva do que seu tratamento quando já instaladas ${ }^{6,12,14}$. A detecção de fatores sociodemográficos, bem como os encargos agravantes aos quais os cuidadores estariam submetidos, permitiriam implementar medidas precoces de prevenção. Assim, o objetivo deste trabalho foi avaliar a freqüência de ansiedade e depressão dos cuidadores informais principais de mulheres na fase terminal de câncer de mama ou genital, bem como identificar algumas características do cuidador que pudessem estar associadas às taxas de ansiedade e depressão detectadas. 
Métodos

\section{Desenho do estudo e seleção dos sujeitos}

Trata-se de estudo de corte transversal, aprovado pelo Comitê de Ética em Pesquisa da Faculdade de Ciências Médicas da UNICAMP. Foram elegiveis 170 pacientes sem possibilidades curativas e acamadas por mais da metade das horas de vigilia, ou incapacitadas, internadas na Enfermaria de Oncologia do Centro de Atenção Integral à Saúde da Mulher (CAISM), Campinas SP, com câncer de mama ou ginecológico.

Após a assinatura do termo de consentimento livre e esclarecido foi realizada breve entrevista semi-estruturada com a paciente ou seu acompanhante, com o objetivo de se obterem dados sobre a idade, o diagnóstico da paciente, o estado civil, a religião e o número de pessoas que viviam na casa. O cuidador principal foi identificado espontaneamente pela própria paciente, e com a ajuda de um familiar nos casos em que a paciente não se encontrava em condições de responder. Trinta e sete pacientes não tiveram seus cuidadores incluídos na pesquisa $(21$ mulheres cujo óbito ocorreu antes da entrevista, 8 em que a paciente não tinha cuidador, 6 em que não foi possivel fazer contato com o cuidador e duas cujos cuidadores optaram por não participar). Assim, neste estudo foram incluídas 133 pacientes com câncer em fase terminal da doença e seus respectivos cuidadores.

\section{Instrumentos de pesquisa}

Os cuidadores informais foram entrevistados na sala de atendimento psicológico ou na sala de espera da enfermaria, por duas psicólogas.

Para avaliar a ansiedade e a depressão foi utilizada a Escala Hospitalar de Ansiedade e Depressão (Hospital Anxiety and Depression Scale, $H A D)$. A HAD é escala que possibilita avaliar separadamente a depressão e a ansiedade tanto em pacientes com outras doenças instaladas como em indivíduos não considerados doentes ${ }^{15}$. É formada por 14 questões, das quais sete relacionadas à ansiedade (HAD-A) e sete à depressão (HAD-D) ${ }^{16}$. O entrevistado atribui nota de zero a três para cada uma das perguntas, perfazendo totais que podem variar de 0 a 21 em cada subescala. Pontuação de oito ou mais, tanto na subescala de ansiedade quanto na de depressão, é sugestiva de transtorno de ansiedade e de depressão, respectivamente ${ }^{17}$, embora a HAD não tenha como objetivo quantificar a gravidade do sintoma. A escala HAD foi validada no Brasil em uma enfermaria de clínica médica ${ }^{18}$.

Após a aplicação do instrumento, todos os cuidadores principais foram submetidos a uma entrevista semi-estruturada para complementação de informações sobre idade, sexo, estado civil, grau de parentesco com a paciente, religião, rodízio familiar, presença de doença do cuidador, emprego e outros encargos familiares como cuidador. As entrevistas duraram, em média, 30 minutos. A HAD foi lida pelo entrevistador para todos os participantes, a fim de contornar problemas de analfabetismo e baixa escolaridade.

\section{Análise estatistica}

A ansiedade e a depressão foram classificadas a partir das escalas HAD-A e HAD-D. Utilizou-se a regressão logística para cálculo do odds ratio (OR) e seus respectivos intervalos de confiança (IC) para avaliar a relação entre os diagnósticos de ansiedade e depressão entre os cuidadores informais, suas características epidemiológicas e suas situações com relação às pacientes. Para a análise múltipla foi considerado o critério de seleção de variáveis passo a passo. Todos os cálculos foram realizados com pacote estatístico SAS versão 8.2. O nivel de significância assumido foi de $5 \%{ }^{19,20}$.

\section{Resultados}

Mais da metade das pacientes apresentava câncer da mama $(53,4 \%)$ e as outras, câncer ginecológico. Mais de um terço das pacientes tinham 50 anos ou menos; $72,9 \%$ apresentavamse acamadas em mais da metade das horas de vigília; $83,5 \%$ estavam conscientes no momento em que seu cuidador foi entrevistado e $56,3 \%$ tiveram sua doença diagnosticada nos 24 meses anteriores à entrevista. Quase metade das pacientes não era casada $(40,6 \%)$ e, entre as casadas, nem todas moravam com seus maridos no momento da entrevista. Outros parentes também moravam na mesma casa em $82,7 \%$ dos casos (Tabela 1). Todas essas características da paciente foram testadas em relação à ansiedade e à depressão do cuidador principal, por meio de regressão logística multivariada. Entretanto, nenhuma variável foi significativa (dados não mostrados em tabelas). 
Tabela 1 - Características clínicas das 133 pacientes.

\begin{tabular}{lrr}
\hline Paciente & $\mathbf{n}$ & $\%$ \\
\hline Tumor Primário & & \\
$\quad$ Câncer ginecológico & 62 & 46,6 \\
$\quad$ Câncer de mama & 71 & 53,4 \\
Idade & & \\
$\quad \leq 50$ anos & 49 & 37,1 \\
$\quad$ >50 anos & 83 & 62,9 \\
Estado físico & & \\
$\quad$ Acamada mais de 50\% das horas de vigília & 97 & 72,9 \\
$\quad$ Incapacitada & 36 & 27,1 \\
Consciente & & \\
$\quad$ Não & 22 & 16,5 \\
$\quad$ Sim & 111 & 83,5 \\
Tempo entre diagnóstico e entrevista & & \\
$\quad \leq 24$ meses & 71 & 56,3 \\
$\quad>24$ meses & 55 & 43,7 \\
Estado conjugal & & \\
$\quad$ Casada & 79 & 59,4 \\
$\quad$ Viúva & 29 & 21,8 \\
Solteira & 14 & 10,5 \\
$\quad$ Divorciada & 11 & 8,3 \\
Religião & & \\
$\quad$ Não & 2 & 1,5 \\
$\quad$ Sim & 131 & 98,5 \\
Mora com marido & & \\
$\quad$ Sim & & \\
$\quad$ Não & 74 & 55,6 \\
Outras pessoas vivem na mesma casa & 59 & 44,4 \\
$\quad$ Não & & \\
$\quad$ Sim & 23 & 17,3 \\
Mora sozinha ou em instituição & 110 & 82,7 \\
$\quad$ Não & & \\
$\quad$ Sim & 127 & 95,5 \\
\hline & 7 & 4,5 \\
\hline
\end{tabular}

Noventa e nove cuidadores principais $(74,4 \%)$ apresentavam ansiedade e $71(53,4 \%)$ apresentavam depressão, quando avaliados segundo a escala HAD. Sessenta e três (89\%) cuidadores informais, que apresentavam depressão, tinham também ansiedade, mostrando que ansiedade e depressão apresentaram grande chance de serem concomitantes (OR=5,6; IC 95\%: 2,2 a 15,9) (Tabela 2).

Tabela 2 - Ansiedade e depressão dos 133 cuidadores principais.

\begin{tabular}{lccc}
\hline Ansiedade & \multicolumn{2}{c}{ Depressão } & OR (IC 95\%) \\
& Sim & Não & \\
\hline Sim & $63(89 \%)$ & $36(58 \%)$ & Ref. \\
Não & $8(11 \%)$ & $26(42 \%)$ & $5,6(2,2-15,9)$ \\
Total & 71 & 62 & \\
\hline
\end{tabular}

A idade dos cuidadores variou de 14 a 79 anos (média 42 anos), sendo que $63 \%$ tinham mais de 35 anos. A maioria dos cuidadores informais era do sexo feminino. Observou-se que 57 pacientes $(43 \%)$ indicaram como cuidador principal a filha e 32 indicaram o marido $(24 \%)$. Praticamente metade (59\%) estava desempregada ou aposentada. Entre os cuidadores informais, $66 \%$ faziam rodízio, ou seja, tinham outras pessoas ajudando a cuidar deste mesmo paciente. Observou-se também que $47 \%$ dos cuidadores dedicavam-se a outra pessoa além da paciente. O óbito da paciente ocorreu em 42,9\% dos casos, em menos de 30 dias após a entrevista com o cuidador e a maioria dos cuidadores $(83 \%)$ referiu mudança na rotina antes do óbito. $\mathrm{Na}$ análise bivariada, apenas o fato de o cuidador ser o marido esteve associado com menor ansiedade, e após regressão logística com seleção passo a passo apenas o fato de ser homem esteve significativamente relacionado com menor proporção de ansiedade entre os cuidadores (Tabelas 3 e 4).

Tabela 3 - Características sociodemográficas e encargos dos cuidadores principais associados à ansiedade.

\begin{tabular}{|c|c|c|c|c|}
\hline Variáveis & $\begin{array}{l}\text { Total } \\
\text { n } \quad(\%)\end{array}$ & $\begin{array}{c}\text { Ansiedade } \\
n \quad(\%)\end{array}$ & $\begin{array}{c}\text { OR (IC } 95 \%) \\
\text { bruto }\end{array}$ & $\begin{array}{c}\text { OR (IC 95\%) } \\
\text { Seleção } \\
\text { passo a } \\
\text { passo } \\
\end{array}$ \\
\hline Idade & & & & - \\
\hline$\leq 35$ & 49 (37) & $39(80)$ & Ref & \\
\hline $36-55$ & $58(44)$ & $44(76)$ & $0,8(0,3$ a 2,0$)$ & \\
\hline$\geq 56$ & $26(19)$ & $16(62)$ & $0,4(0,1$ a 1,2$)$ & \\
\hline \multicolumn{5}{|l|}{ Sexo } \\
\hline Feminino & $90(68)$ & $74(82)$ & Ref & \\
\hline Masculino & $43(32)$ & $25(58)$ & $0,3(0,1$ a 0,7$)$ & $0,3(0,1$ a 0,7$)$ \\
\hline Parentesco & & & & - \\
\hline Marido & $32(24)$ & $17(53)$ & Ref & \\
\hline Filha & $57(43)$ & $46(81)$ & $3,7(1,4$ a 9,6$)$ & \\
\hline Outros* & $44(33)$ & $36(82)$ & $4,0(1,4$ a 11,2$)$ & \\
\hline Ocupação & & & & - \\
\hline Empregados & $55(41)$ & $45(82)$ & Ref & \\
\hline Não empregados** & $52(39)$ & $38(73)$ & $0,6(0,2$ a 1,2$)$ & \\
\hline Aposentados & $26(20)$ & $16(62)$ & $0,4 \quad(0,1$ a 1,0$)$ & \\
\hline Rodízio & & & & - \\
\hline Não & $45(34)$ & $35(78)$ & Ref & \\
\hline $\operatorname{Sim}$ & $88(66)$ & $64(73)$ & $0,8 \quad(0,3$ a 1,8$)$ & \\
\hline Cuida de outras & & & & - \\
\hline \multicolumn{5}{|l|}{ pessoas } \\
\hline Não & $71(53)$ & $50(70)$ & Ref & \\
\hline $\operatorname{Sim}$ & $62(47)$ & $49(79)$ & $1,6(0,7$ a 3,5$)$ & \\
\hline $\begin{array}{l}\text { Tempo entre } \\
\text { entrevista e óbito }\end{array}$ & & & & - \\
\hline$\leq 30$ dias & $62(47)$ & $50(81)$ & Ref & \\
\hline$>30$ dias & 71 (53) & 49 (69) & $0,5(0,2$ a 1,2$)$ & \\
\hline Mudou a rotina & & & & - \\
\hline Sim & $111(84)$ & $84(76)$ & Ref & \\
\hline Não & $22(16)$ & $15(68)$ & $1,5(0,5$ a 3,9$)$ & \\
\hline
\end{tabular}


Tabela 4 - Características sociodemográficas e encargos dos cuidadores principais associados à depressão.

\begin{tabular}{|c|c|c|c|c|c|c|}
\hline \multirow[t]{2}{*}{ Variáveis } & \multicolumn{2}{|c|}{ Total } & \multicolumn{2}{|c|}{ Ansiedade } & \multirow{2}{*}{\multicolumn{2}{|c|}{$\begin{array}{c}\text { OR (IC 95\%) } \\
\text { bruto }\end{array}$}} \\
\hline & $\mathrm{n}$ & $(\%)$ & $\mathbf{n}$ & $(\%)$ & & \\
\hline \multicolumn{7}{|l|}{ Idade } \\
\hline$\leq 35$ & 49 & (37) & 24 & (49) & Ref & \\
\hline $36-55$ & 58 & (44) & 35 & (60) & 1,6 & $(0,7$ a 3,4$)$ \\
\hline$\geq 56$ & 26 & (19) & 12 & (46) & 0,9 & $(0,3$ a 2,3$)$ \\
\hline \multicolumn{7}{|l|}{ Sexo } \\
\hline Feminino & 90 & (68) & 53 & (59) & Ref & \\
\hline Masculino & 43 & (32) & 18 & (42) & 0,5 & $(0,2$ a 1,0$)$ \\
\hline \multicolumn{7}{|l|}{ Parentesco } \\
\hline Marido & 32 & (24) & 14 & (44) & Ref & \\
\hline Filha & 57 & (43) & 35 & (61) & 1,9 & $(0,9$ a 4,2$)$ \\
\hline Outros* & 44 & (33) & 22 & (50) & 1,0 & $(0,4$ a 2,4$)$ \\
\hline \multicolumn{7}{|l|}{ Ocupação } \\
\hline Empregados & 55 & (41) & 26 & $(47)$ & Ref & \\
\hline Não empregados** & 52 & (39) & 33 & (63) & 1,9 & $(0,9$ a 4,2$)$ \\
\hline Aposentados & 26 & (20) & 12 & $(46)$ & 1,0 & $(0,4$ a 2,4$)$ \\
\hline \multicolumn{7}{|l|}{ Rodízio } \\
\hline Não & 45 & (34) & 24 & (53) & Ref & \\
\hline Sim & 88 & $(66)$ & 47 & (53) & 1,0 & $(0,5$ a 2,1$)$ \\
\hline \multicolumn{7}{|l|}{$\begin{array}{l}\text { Cuida de outras } \\
\text { pessoas }\end{array}$} \\
\hline Não & 71 & (53) & 37 & $(52)$ & Ref & \\
\hline Sim & 62 & $(47)$ & 34 & $(55)$ & 1,1 & $(0,6$ a 2,2$)$ \\
\hline \multicolumn{7}{|l|}{$\begin{array}{l}\text { Tempo entre } \\
\text { entrevista e óbito }\end{array}$} \\
\hline$\leq 30$ dias & 62 & $(47)$ & 38 & (61) & Ref & \\
\hline$>30$ dias & 71 & (53) & 33 & (46) & 0,6 & $(0,3$ a 1,1) \\
\hline \multicolumn{7}{|l|}{ Mudou a rotina } \\
\hline Sim & 111 & (84) & 62 & $(56)$ & Ref & \\
\hline Não & 22 & (16) & 9 & (41) & 1,8 & $(0,7$ a 4,6$)$ \\
\hline
\end{tabular}

*Outros: filho (9), irmã (9), mãe (9), vários (17).

**Dona de casa (28), estudante (8), desempregado (24).

\section{Discussão}

O estudo revelou que considerável proporção dos cuidadores informais principais de pacientes com câncer de mama ou ginecológico, na fase terminal da doença, apresentam ansiedade e depressão. Existe inter-relacionamento entre o sofrimento psíquico da família e o sofrimento do paciente, com conseqüências diretas sobre ambos. A depressão e a ansiedade no cuidador podem desencadear depressão e ansiedade no paciente e vice-versa; ou seja, se a família é afetada pela doença, as reações familiares também afetam o paciente. Tais evidências reforçam a idéia de que o processo de cuidar é bastante complexo, influenciando e sendo influenciado pelo contexto do adoecer e do morrer ${ }^{2,6,21}$. Assim, é justificável a preocupação crescente em estudar esse impacto. Avaliar e tratar a ansiedade e a depressão no cuidador informal tem importante valor preventivo ${ }^{2,3}$. O sin- toma de ansiedade detectado neste estudo foi muito elevado, sendo observado em mais de $70 \%$ dos casos, e o de depressão em mais da metade. Estes dados são superiores às médias de $30 \%$ para depressão e $45 \%$ para ansiedade, descritas na literatura ${ }^{6,9}$.

No presente estudo, as características da paciente (idade, estado físico, diagnóstico) não estiveram associadas à ansiedade e depressão do cuidador principal. Neste, os altos índices de ansiedade e depressão ocorreram independentemente das características sociodemográficas da paciente e do cuidador, bem como de seus encargos; se ele contava com ajuda de outras pessoas para cuidar; se fazia rodízio; se cuidava de outras pessoas além da paciente ou da proximidade do óbito. Após regressão logística, apenas o fato de o cuidador ser do sexo masculino esteve associado a menor freqüência de ansiedade.

Observou-se neste estudo que a maioria dos cuidadores era constituída de mulheres jovens, geralmente a filha, que representou quase a metade da população total de cuidadores, seguida da irmã, mãe, cunhada, amiga, nora, sobrinha, tia e neta. Nos cuidadores do sexo masculino, o marido foi o tipo de parentesco mais freqüente, seguido do filho, irmão e genro. Estes resultados também são encontrados na literatura, que descreve os cuidadores de paciente com câncer de mama ou genital, em geral, como mulheres adultas ou cônjuges $^{22-24}$.

No grupo feminino verificou-se alto índice de ansiedade e de depressão. Em estudo sobre as necessidades de suporte para o cuidador informal de pacientes com câncer, ser mais jovem e mulher estava relacionado com alto nivel de morbidade psicológica e tensão ${ }^{25}$. Além disso, o fato de ser mulher e filha pode provocar, pelo processo de identificação com a paciente, grande variedade de sentimentos. Outros autores descrevem que as famílias que precisaram de maior apoio emocional foram aquelas em que os pacientes eram jovens, mulheres com diagnóstico de câncer de mama ou que estavam sofrendo sintomas físicos graves ${ }^{6}$.

No presente estudo quando o homem assumiu o papel de cuidador, em geral o marido, observou-se maior tendência à aceitação desta função. $\mathrm{O}$ fato de ser marido da paciente esteve associado com menor ansiedade apenas na análise bivariada, e a depressão foi menor entre os homens, embora com valores não significativos. Estudos demonstram que homens, avaliados em momentos de pós-estresse traumático, produzem serotonina mais rapidamente, quando comparados com mulheres. Como a serotonina influencia o humor, esta des- 
coberta pode ajudar a explicar porque muito mais mulheres do que homens sofrem de depressão ${ }^{26}$. Entretanto, alguns aspectos práticos precisam ser considerados: a enfermaria de oncologia do CAISM é composta por quartos onde são internadas até três mulheres com câncer de mama ou ginecológico. Os acompanhantes e cuidadores podem permanecer com seu paciente durante a maior parte do tempo, porém, não há instalações que facilitem a permanência de homens.

A qualidade da relação entre paciente e cuidador é considerada importante fator na mediação do estresse e, indiretamente, contribui para a redução do impacto causado pela doença no cuidador. Quando os cuidadores avaliam a relação de forma positiva, eles reportam menos impacto e menos sintomas relacionados a estresse e depressão. Somado a isto, a convivência da filha com uma doença que acomete a mulher de forma indiscriminada e com alta prevalência pode intensificar os temores e sentimentos de ameaça a si. Na relação mãe-filha aparecem as fantasias relacionadas à herança genética, cada vez mais discutida socialmente, trazendo carga de ansiedade adicional a esta população ${ }^{27}$.

Nos estudos que abordam as conseqüências de cuidar do paciente nesta fase, a experiência é descrita como muito pesada e negativa para os cuidadores informais ${ }^{1,3,5}$. Porém, essas conseqüências costumam ser subestimadas, talvez pela falta de conhecimento especializado das doenças psicológicas ou pela visão equivocada de que a depressão é "normal" entre pacientes que estão morrendo e em seus cuidadores ${ }^{12,14,21,28}$. Para compreender melhor o impacto do cuidar na fase terminal da doença, seriam importantes mais duas avaliações da ansiedade e da depressão do cuidador: uma antes desta fase e outra após a ocorrência do óbito. Estas serviriam de parâmetros para comparar a evolução do quadro psicológico: se de fato se intensificaram na fase terminal e/ou se alteraram após o óbito da paciente.

Ao demonstrar que as características objetivas do cuidador pouco estiveram associadas à depressão e à ansiedade, este trabalho apontou também para a necessidade de um instrumento complementar, multidimensional, padronizado e adequado para o contexto do cuidar na fase terminal, com a finalidade de detectar mais fatores associados, tais como presenciar o sofrimento físico e psíquico do paciente na iminência da morte, nos aspectos subjetivos, positivos e negativos, implícitos neste processo.

Concluímos com este estudo que existe alto indice e concomitância de ansiedade e depressão na população de cuidadores principais de pacien- tes com câncer de mama ou ginecológico na fase terminal. Observamos também que a idade do cuidador, o parentesco com a paciente, o estado conjugal, a ocupação profissional, se recebe ajuda de outras pessoas para cuidar deste paciente ou outras dificuldades do cuidador não estavam associados à ansiedade ou à depressão, e que apenas o fato de o cuidador ser homem e marido da paciente associou-se a menor freqüência de ansiedade. No entanto, o processo de cuidar de um ente querido na fase terminal é em si mesmo fator bastante estressante, e outras variáveis associadas ao impacto mental do cuidar necessitam ser investigadas.

\section{Referências}

1. Klinkenberg M, Willems DL, van der Wal G, Deeg DJ. Symptom burden in the last week of life. J Pain Symptom Manage. 2004;27(1):5-13.

2. Armes PJ, Addington-Hall JM. Perspectives on symptom control in patients receiving community palliative care. Palliat Med. 2003;17(7):608-15.

3. Wong RK, Franssen E, Szumacher E, Connolly R, Evans M, Page B, et al. What do patients living with advanced cancer and their carers want to know? - a needs assessment. Support Care Cancer. 2002;10(5):408-15.

4. World Health Organization. Cancer pain relief and palliative care. Geneva: WHO; 1990.

5. Rabow MW, Hauser JM, Adams J. Supporting family caregivers at the end of life: "they don't know what they don't know". JAMA. 2004;291(4):483-91.

6. Hodgson C, Higginson I, McDonnel M, Butters E. Family anxiety in advanced cancer: a multicentre prospective study in Ireland. $\mathrm{Br} \mathrm{J}$ Cancer. 1997;76(9):1211-4.

7. Deeken JF, Taylor KL, Mangan P, Yabroff KR, Ingham JM. Care for the caregivers: a review of self-report instruments developed to measure the burden, needs, and quality of life of informal caregivers. J Pain Symptom Manage. 2003;26(4):922-53.

8. Cella DF, Bonomi AE. Measuring quality of life: 1995 update. Oncology (Williston Park). 1995;9(11 Suppl):47-60.

9. Grov EK, Dahl AA, Moum T, Fossa SD. Anxiety, depression, and quality of life in caregivers of patients with cancer in late palliative phase. Ann Oncol. 2005;16(7):1185-91.

10. Grunfeld E, Coyle D, Whelan T, Clinch J, Reyno L, Earle CC, et al. Family caregiver burden: results of a longitudinal study of breast cancer patients and their principal caregivers. CMAJ. 2004;170(12):1795-801. 
11. Butler LD, Field NP, Busch AL, Seplaki JE, Hastings TA, Spiegel D. Anticipating loss and other temporal stressors predict traumatic stress symptoms among partners of metastatic/recurrent breast cancer patients. Psychooncology. 2005;14(6):492-502.

12. Jones RD. Depression and anxiety in oncology: the oncologist's perspective. J Clin Psychiatry. 2001;62 Suppl 8:52-5.

13. Thompson C. Affective disorders. In: Thompson C, editor. The instruments of psychiatric research. London: John Wiley \& Sons; 1989. p. 87-126.

14. Higginson I, Priest P. Predictors of family anxiety in the weeks before bereavement. Soc Sci Med. 1996;43(11):1621-5.

15. Ballenger CJ, Davidson JR, Lecrubier Y, Nutt DJ, Jones RD, Berard RM, et al. Consensus statement on depression, anxiety, and oncology. J Clin Psychiatry. 2001;62 Suppl 8:64-7.

16.Zigmond AS, Snaith RP. The hospital anxiety and depression scale. Acta Psychiatr Scand. 1983;67(6):361-70.

17. Botega NJ, Pondé MP, Medeiros P, Lima MG, Guerreiro CAM. Validação da escala hospitalar de ansiedade e depressão (HAD) em pacientes epiléticos ambulatoriais. J Bras Psiquiatr. 1998;47(6):258-89.

18. Botega NJ, Bio MR, Zomignani MA, Garcia Junior C, Pereira WAB. Transtornos do humor em enfermaria de clínica médica e validação de escala de medida (HAD) de ansiedade e depressão. Rev Saúde Pública. 1995;29(5):355-63.

19. Altman DG. Pratical statistics for medical research. London: Chapman and Hall; 1991.

20.SAS/STAT Software. Changes and enhancement through release 8.2 [computer program]. Cary: SAS Institute; 1999-2001.
21.Kurtz ME, Kurtz JC, Given CW, Given B. Relationship of caregiver reactions and depression to cancer patients' symptoms, functional states and depression: a longitudinal view. Soc Sci Med. 1995;40(6):837-46.

22. Harrigton V, Lackey NR, Gates MF. Needs of caregivers of clinic and hospice cancer patients. Cancer Nurs. 1996;19(2):118-25.

23. Kristjanson LJ, Leis A, Koop PM, Carrière KC, Mueller B. Family Members' care expectations, care perceptions and satisfaction with advanced cancer care: results of a multi-site pilot study. J Palliat Care. 1997;13(4):5-13.

24. Nijboer C, Triemstra M, Tempelaar R, Sanderman $\mathrm{R}$, van den Bos GA. Measuring both negative and positive reactions to giving care to cancer patients: psychometric qualities of the Caregiver Reaction Assessment (CRA). Soc Sci Med. 1999;48(9):125969.

25. Payne S, Smith P, Dean S. Identifying the concerns of informal carers in palliative care. Palliative Med. 1999;13(1):37-44.

26. Pasacreta JV, Barg F, Nuamah I, McCorkle R. Participant characterisitics before and 4 months after attendence at a family caregiver cancer education program. Cancer Nurs. 2000;23(4):295303.

27.Kessler S. Psychological aspects of genetic counseling: VI. A critical review of the literature dealing with education and reproduction. Am J Med Genet. 1989;34(3):340-53.

28. Emanuel EJ, Emanuel LL. The promise of a good death. Lancet. 1998;351 Suppl 2:SII21-9. 